\title{
ARTICLE Efficacy of PARP inhibition in Pde6a mutant mouse models for retinitis pigmentosa depends on the quality and composition of individual human mutations
}

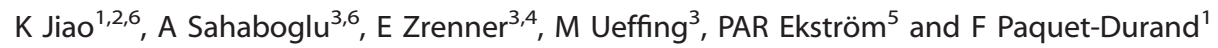

Retinitis pigmentosa (RP), an inherited blinding disease, is caused by a variety of different mutations that affect retinal photoreceptor function and survival. So far there is neither effective treatment nor cure. We have previously shown that poly(ADPribose)polymerase (PARP) acts as a common and critical denominator of cell death in photoreceptors, qualifying it as a potential target for future therapeutic intervention. A significant fraction of RP-causing mutations affect the genes for the rod photoreceptor phosphodiesterase 6A (PDE6A) subunit, but it is not known whether they all engage the same death pathway. Analysing three homozygous point mutations (Pde6a R562W, D670G, and V685M) and one compound heterozygous Pde6a ${ }^{V 685 M / R 562 W}$ mutation in mouse models that match human RP patients, we demonstrate excessive activation of PARP, which correlated in time with the progression of photoreceptor degeneration. The causal involvement of PARP activity in the neurodegenerative process was confirmed in organotypic retinal explant cultures treated with the PARP-selective inhibitor PJ34, using different treatment timepoints and durations. Remarkably, the neuroprotective efficacy of PARP inhibition correlated inversely with the strength of the genetically induced insult, with the D670G mutant showing the best treatment effects. Our results highlight PARP as a target for neuroprotective interventions in RP caused by PDE6A mutations and are a first attempt towards personalized, genotype-matched therapy development for RP. In addition, for each of the different mutant situations, our work identifies windows of opportunity for an optimal treatment regimen for further in vivo experimentation and possibly clinical studies.

Cell Death Discovery (2016) 2, 16040; doi:10.1038/cddiscovery.2016.40; published online 4 July 2016

\section{INTRODUCTION}

Retinitis pigmentosa (RP) is a hereditary disease that causes the progressive degeneration and death of photoreceptors and is one of the main causes of blindness in the developed world, ${ }^{1}$ affecting $\sim 1$ in 4000 people. $^{2} \mathrm{RP}$ is genetically and clinically heterogeneous, with onset varying from early childhood to late adulthood depending on the exact mutation and, probably, other factors. Mutations in more than 60 genes are associated with RP (https://sph.uth.edu/retnet), giving a complexity that may require personalized therapy approaches, which in turn necessitates knowledge on the pathology of the individual mutations. At the same time, there is a need to identify common principles that can be exploited for the development of therapies addressing many different types of mutations at once.

As many as $10 \%$ of human RP patients may suffer from mutations in one of the three genes encoding for phosphodiesterase 6 (PDE6). ${ }^{3-5}$ In rod photoreceptors, PDE6 consists of two catalytic subunits $\alpha$ and $\beta$ (A and $B$ ) and an inhibitory $\gamma(G)$ subunit and has the function to hydrolyse cGMP in response to light. Although each of the three PDE6 subunits can be affected by mutations, previous research on Pde6 mutant animals has mostly focussed on Pde6b mutants, namely the $r d 1$ and $r d 10$ mouse models. ${ }^{6,7}$ An excessive activation of poly(ADP-ribose)polymerase
(PARP) has been shown to not only have an important role in many neurodegenerative diseases but it may also contribute to caspase-independent photoreceptor cell death. ${ }^{8,9}$ Interestingly, in the Pde6b mutants, PARP activity was shown to be involved in the progression of photoreceptor degeneration, and PARP appears also to directly participate in photoreceptor degeneration in models with unrelated mutations. ${ }^{10,11}$ PARP therefore has the quality of a common denominator in photoreceptor degeneration, and is, as such, an interesting molecular target, although mutation-dependent aspects may very well occur. ${ }^{11}$ To date, Pde6a mutants have been far less studied and it is not clear if they adhere to such a PARP involvement, nor if the possible characteristics of the latter will make it available for neuroprotective intervention.

At least 29 different PDE6A mutations are known from RP patients (the human gene mutation database; http://www.hgmd. cf.ac.uk; information retrieved July 2015); thus, to understand the degenerative events in the human situation, it is necessary to come as close as possible to the defined genetic defects when we select our study material. Here, we used three different homozygous Pde6a mutant mice, Pde6a ${ }^{R 562 W}, P_{\text {de } 6 a^{D 670 G} \text {, and }}$ Pde6a ${ }^{V 685 M}$, as well as compound heterozygous Pde6a $a^{V 685 M^{\prime} R 562 W}$ animals. Importantly, genotypes homologous to the homozygous

\footnotetext{
${ }^{1}$ Cell Death Mechanisms Group, Division of Experimental Ophthalmology, Centre for Ophthalmology, Institute for Ophthalmic Research, University of Tuebingen, Roentgenweg 11, Tuebingen 72076, Germany; ${ }^{2}$ Centre for Ophthalmology, The Second People's Hospital of Yunnan Province and The Fourth Affiliated Hospital of Kunming Medical University, Qingnian 176, Kunming 650021, China; ${ }^{3}$ Centre for Ophthalmology, Institute for Ophthalmic Research, University of Tuebingen, Tuebingen 72076 , Germany; ${ }^{4}$ Werner Reichardt

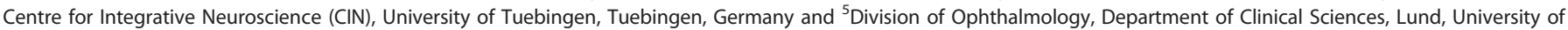
Lund, Lund 22184, Sweden.

Correspondence: A Sahaboglu (ayse.sahaboglu-tekgoez@klinikum.uni-tuebingen.de) or F Paquet-Durand (francois.paquet-durand@klinikum.uni-tuebingen.de)

${ }^{6}$ These authors contributed equally to this work presented here and should therefore be regarded as equivalent first authors.
}

Received 7 April 2016; accepted 28 April 2016; Edited by R Killick 
Pde6 $a^{\mathrm{V} 685 \mathrm{M} / \mathrm{V} 685 \mathrm{M}}$ and the compound heterozygous Pde6a ${ }^{\mathrm{V} 685 \mathrm{M}^{*} \mathrm{R} 562 \mathrm{~W}}$ mouse mutant, respectively, are present among RP patients. ${ }^{12,13}$ This therefore provided us with the rare opportunity to attempt a personalized medicine approach, studying two RP mouse models genotype-matched to human RP, both with respect to the mechanistic components and to how they respond to a defined treatment. The two additional mutations further increased the insight into how alternative mutations in the same gene can affect such parameters. For the sake of brevity, in the following, we refer to the Pde6a mutant animals as V685M, V685M*R562W, R562W, and D670G, respectively.

Using these homozygous and compound heterozygous Pde6a mutant animals, we show that PARP activity during photoreceptor neurodegeneration is raised upon Pde6a deficiency. Conversely, in vitro based tests indicate that the selective inhibition of PARP reduces photoreceptor cell death, an effect that was found dependent on the severity of the genetically induced insult and the time-point of therapeutic intervention. Our study thus suggests the likelihood of individual onset and progression patterns in human RP patients associated with different mutations. The use of rodent disease models, matched to individual patients, can therefore support preclinical pharmacological therapy development for RP that reflects the genetic heterogeneity in the human condition.

\section{RESULTS}

PARP activity is increased in Pde6a mutants, concomitant with cell death

Previous studies showed that PARP activity and the accumulation of its product poly(ADP-ribose) was increased during photoreceptor degeneration in the Pde6b mutant $r d 1$ mouse retina. ${ }^{10}$ Hereafter, we refer to poly(ADP-ribose) as PAR and to the process as PARylation. We first performed an in situ PARP activity assay to analyse the temporal appearance of PARP activity in the photoreceptor layer in Pde6a mutants. The number of photoreceptor cells showing high PARP activity was quantified and peak activities were found at P12, $\mathrm{P} 13, \mathrm{P} 15$, and $\mathrm{P} 21$ for the V685M, V685M*R562W, R562W, and D670G mutants, respectively (Figures $1 \mathrm{a}-\mathrm{d}$ ).

At the respective peaks of PARP activity, a statistical analysis was performed, and in all Pde6a mutant genotypes the number of PARP activity-positive cells was significantly higher compared with that in the corresponding wild-type (wt) cells (Figures 1a-d). Previously, we had assessed the progression of photoreceptor degeneration in the various Pde6a mutants, using the terminal deoxynucleotidyl transferase dUTP nick-end labelling (TUNEL) assay to label dying cells in the outer nuclear layer (ONL) at different postnatal ages. ${ }^{13}$ These data showed peaks of cell death at P12, P15, P15, and P21 for the V685M, V685M*R562W, R562W, and D670G mutants, respectively, and is reproduced here for comparison only. We consider the occurrence of the peak of cell death to correlate with the progression of retinal degeneration such that the V685M mutant with its early peak is the most rapidly degenerating model of the four.

In all these situations, the peak of PARP activity in principle coincided in time with the peak of photoreceptor cell death. The relative proportion of cells showing PARP activity (i.e. the peak height) was, however, different in the various genotypes. In the very rapidly degenerating $\mathrm{V} 685 \mathrm{M}$ mutant, at the peak of cell death, only $\sim 1.4 \%$ of cells displayed high PARP activity. This corresponded to $20 \%$ of dying cells. In the more slowly degenerating $\mathrm{V} 685 \mathrm{M} * \mathrm{R} 562 \mathrm{~W}$ and $\mathrm{R} 562 \mathrm{~W}$ mutants, the proportion of PARP activity-positive cells versus dying cells was higher, whereas in the D670G mutant, with the slowest progression of retinal degeneration, the number of cells showing high PARP activity was essentially the same (i.e. 100\%) as the number of dying cells.
Excessive PARP activity may lead to an accumulation of its product PAR, which can be studied using immunohistochemistry. ${ }^{14}$ This staining suggested increased PARylation in the photoreceptor layer in each Pde6a mutant (Figures 1e-h). Quantification and statistical analysis demonstrated significant increases in the number of PAR-positive cells in all Pde6a mutants at the respective peak of degeneration, providing an independent confirmation of the excessive activation of PARP in dying photoreceptors.

PARP inhibition delays photoreceptor cell death in PDE6a mutants The number of TUNEL- and PARP-positive cells in the ONL of Pde6a mutants was strongly increased when compared with wt cells. In addition, the appearance of cell death and PARP activity was highly correlated in time, suggesting a causal relationship between the two. As inhibition of PARP can protect the photoreceptors of the cultured $r d 1$ retina, ${ }^{10}$ we hypothesized that Pde6a mutants would likewise be helped by such intervention.

Retinal explants from Pde6a mutants were cultured and treated with $6 \mu \mathrm{M}$ PJ34, a well-known inhibitor of PARP that is effective on degenerating photoreceptors at this concentration. ${ }^{10}$ To reveal the effective treatment period for neuroprotection, we tested this compound in both short- and long-term approaches. First, we applied a short-term culture paradigm, which consisted of starting the cultures when the mice were at P5 and finishing them when they were at a stage corresponding to P15. Following 4 days in vitro (DIV) of adaptation, the cultures were treated with PJ34, from P9 until P15 (i.e. P5+4+6 DIV; see Material and Methods), after which the TUNEL assay was used to quantitate photoreceptor cell death. In addition, counting the number of ONL cell rows was used to assess the photoreceptor survival.

All Pde6a mutants demonstrated high levels of TUNEL positivity in the untreated groups (Figure 2). However, PARP inhibition with PJ34 significantly decreased the percentages of dying photoreceptors in all four Pde6a mutants (Figure 2), suggesting that these mutants responded to PARP inhibition in a favourable way. Interestingly, even though the basal TUNEL value for untreated wt explants was low (about 1\%), treatment with PJ34 was still able to reduce this in a significant manner, indicating that also the stress evoked by the explantation and culturing lead the photoreceptors into a PARP-dependent death pathway. Conversely, when the treatment effects were analysed as the number of surviving rows of photoreceptors, rather than the number of dying cells, there was a very clear indication that the TUNEL reduction translated into increased photoreceptor survival, as all treated mutant explants had an average cell row number that was higher compared with that in their untreated counterparts. Moreover, even within the short time frame of this paradigm (treatment from P9 to P15), PJ34 treatment resulted in significantly higher values for surviving cell rows in $\mathrm{V}_{685} \mathrm{M}^{*} \mathrm{R} 562 \mathrm{~W}, \mathrm{R} 562 \mathrm{~W}$, and D670G explants (Figure 2).

\section{PARP inhibition decreases PAR accumulation in Pde6a mutant} retina

To confirm that the short-term effects of PJ34 were indeed related to PARP inhibition, we analysed if the PJ34 treatment led to any reduced PARP activity as expressed by the formation of PAR polymers, or PARylation. As shown in Figure 1, PAR-positive cells were identified in the ONL of all Pde6a mutants and the number of PAR-positive cells was clearly reduced by PJ34 treatment (Figure 3). Although this effect was visible even in wt explant cultures, the reduction of ONL cells displaying PAR accumulation was most pronounced in the more slowly degenerating R562W and D670G mutants. These results thus indicated that PJ34 treatment indeed had strongly reduced the PARP activity in retinal photoreceptors. 

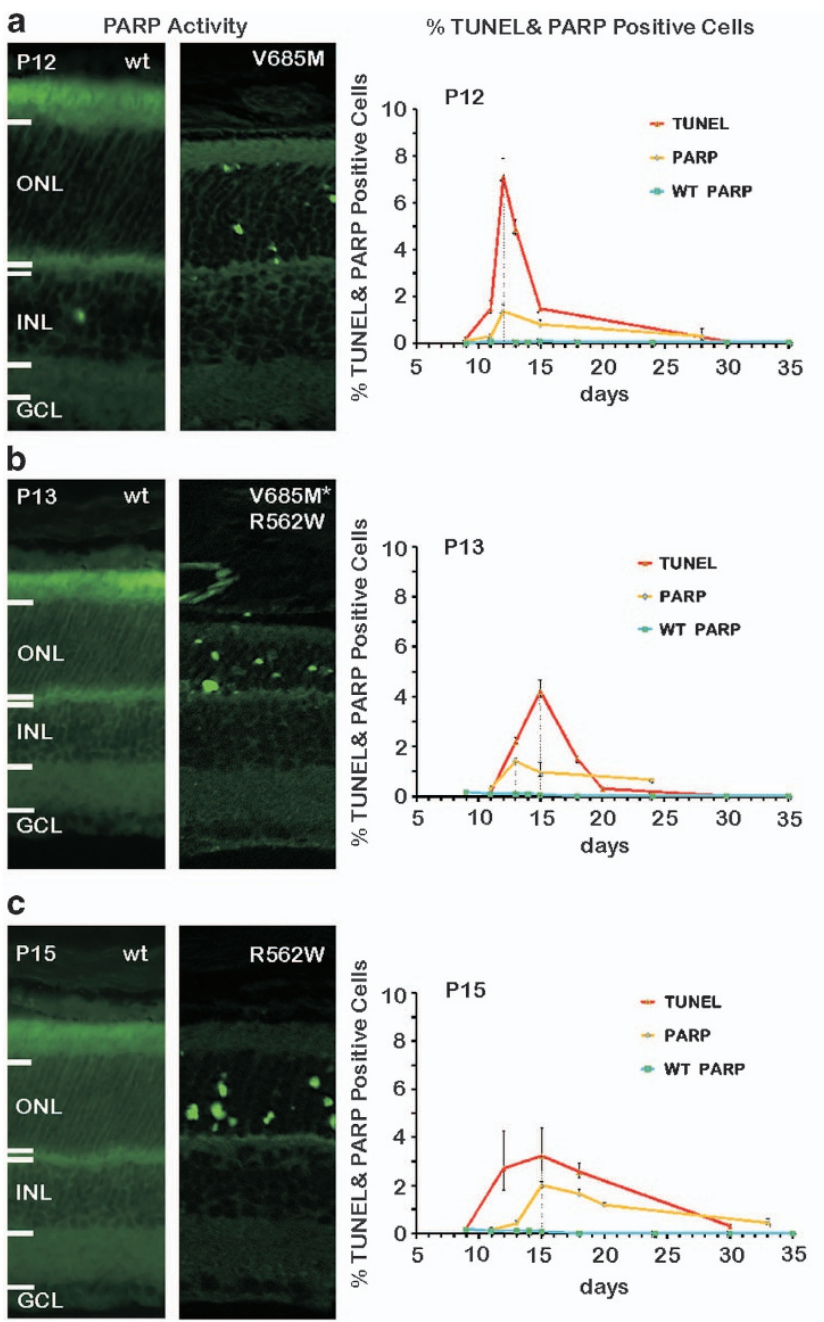

d
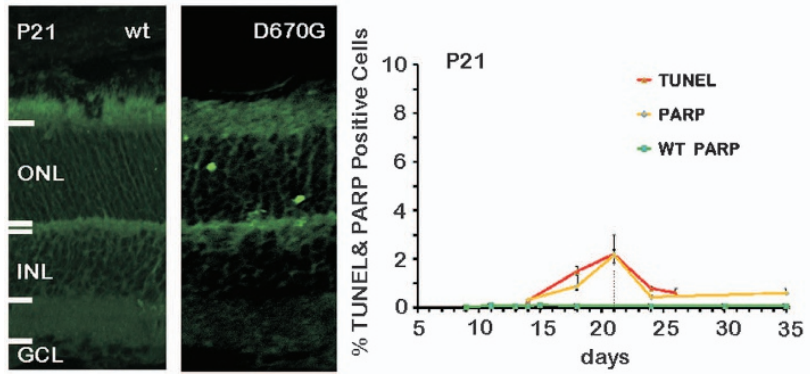
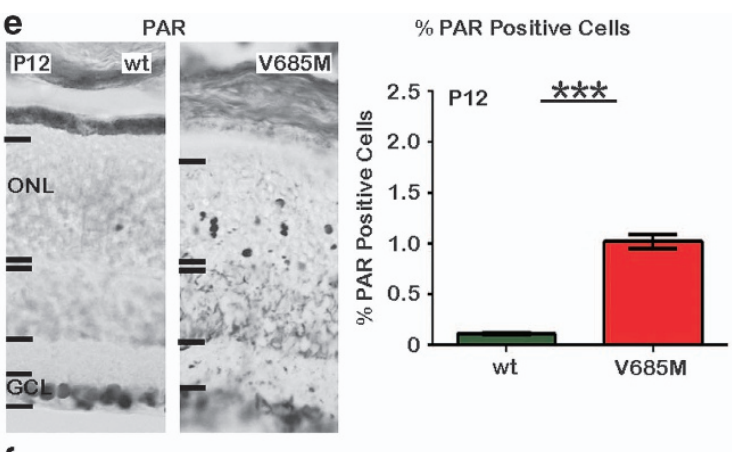

f
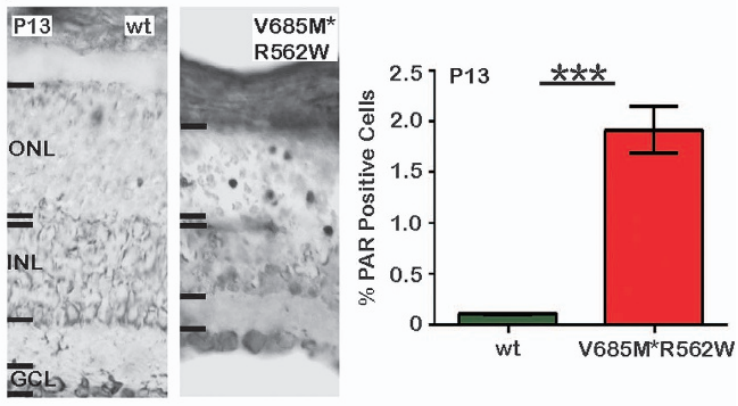

\section{g}
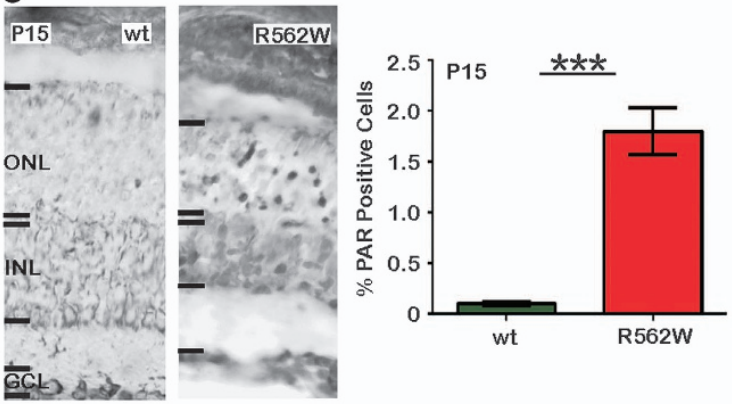

h
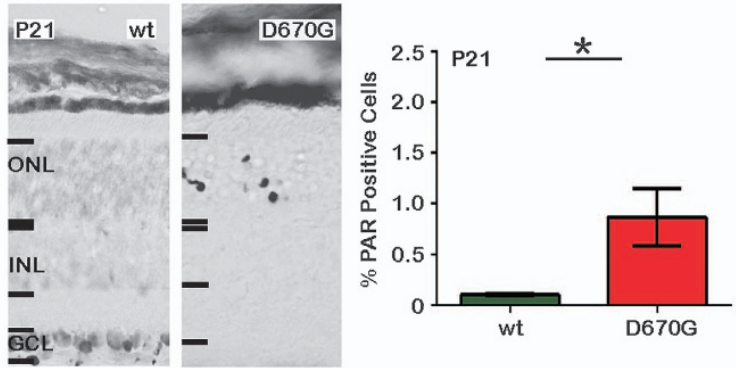

Figure 1. PARP activity and PARylation in Pde6a mutant photoreceptors. The number of PARP activity-positive cells in the V685M, V685M*R562W, R562W, and D670G photoreceptors was strongly increased when compared with wt cells. The quantification of PARP activitypositive cells during the first 30 postnatal days (orange curve) identified peaks of activity at P12, P13, P15, and P21 in the V685M, V685M*R562W, R562W, and D670G mutants, respectively. Asterisks indicate significant levels of mutant versus wt PARP activity. A comparison with the number of dying, TUNEL-positive cells (red curve) in three out of four mutations showed a strong correlation with the peaks of PARP activity, while in the V685M*R562W model, PARP activity appeared to precede cell death (a-d). Similarly, a staining for the accumulation of PARylated proteins - products of PARP activity - showed a strong increase in all four Pde6a mutants when compared with wt cells. At the respective peaks of PARP activity, the number of PAR-positive cells was significantly increased in all four mutants (e-h). The images shown are representative for observations on at least six different specimens for each genotype. Values shown in line and bar graphs are mean \pm S.E.M., with $n=6$ in all cases. For easy reference, the peak time-points of PARP activity are stated in each such graph (a-d).

Long-term retinal explant cultures delineate windows of opportunity

When the culturing period was prolonged from P15 to P19, and thus the treatment extended to 10 DIV (P9-P19), PJ34 replicated very well its actions in the shorter term. As described previously, PJ34 had a significant positive effect on the number of surviving photoreceptor rows in all models except the V685M mutant (Supplementary Figure S1). In fact, the effects as such may even be seen as proportionally higher with the longer treatment, at least for the V685M*R562W and D670G mutants, since in these PJ34 now about doubled the number of remaining rows. We also note that in the untreated V685M, V685M*R562W, and R562W mutants, 

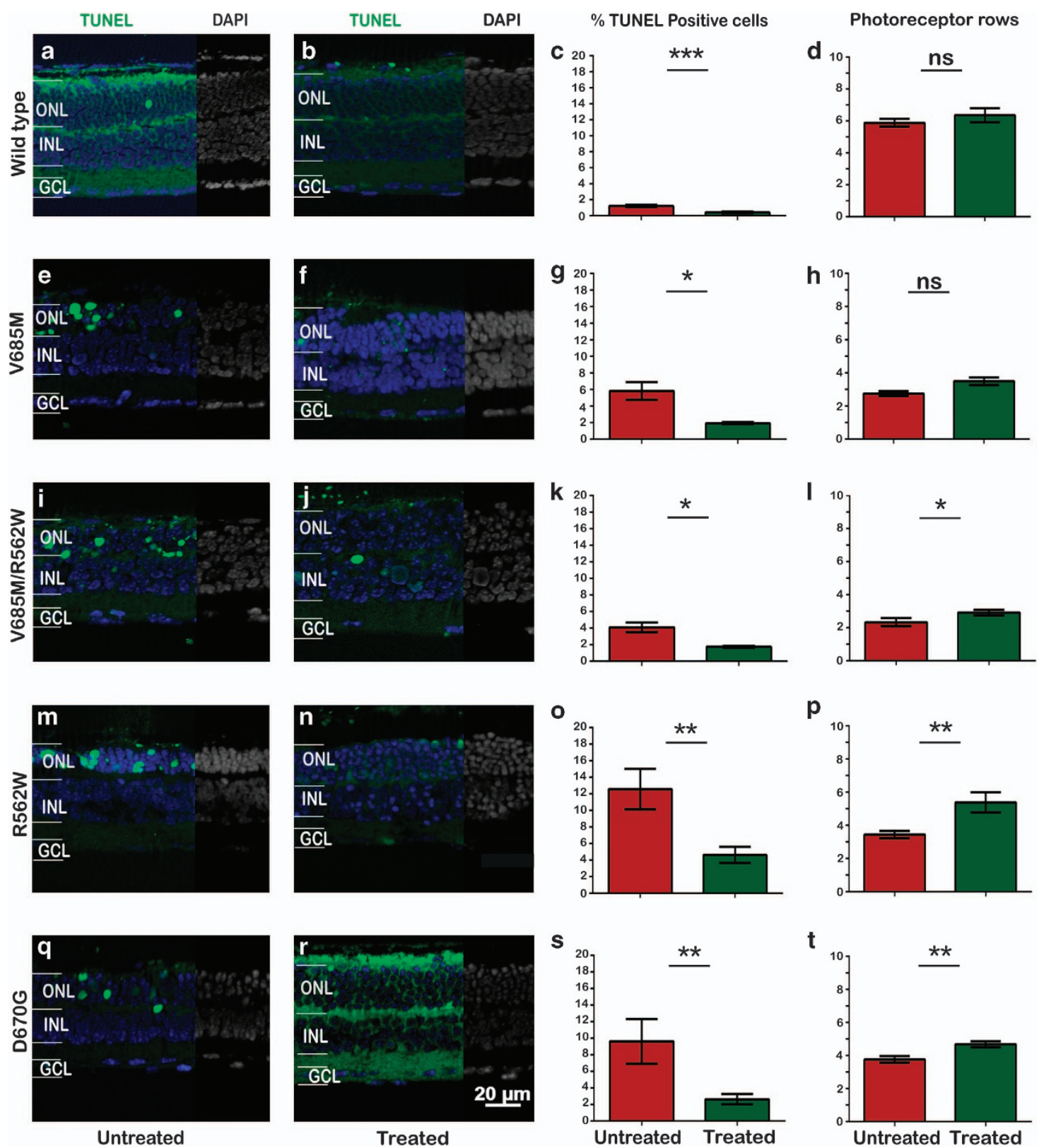

Figure 2. PARP inhibition delays photoreceptor degeneration in Pde6a mutants. In short-term retinal explant cultures (P5-P15; i.e. P5+10 DIV, of which the last 6 days were with treatment), the number of dying, TUNEL-positive cells (green) in the ONL of wt retina (a and b) was significantly reduced by treatment with PJ34 (c), whereas the number of surviving photoreceptor rows remained unchanged (d). In the rapidly degenerating V685M mutant, at P15, PJ34 reduced the amount of cell death without significantly increasing photoreceptor survival (e-h). In the more slowly degenerating V685M*R562W (i-I), R562W (m-p), and D670G retinas $(\mathbf{q}-\mathbf{u})$, a reduction of cell death lead to significant increases in photoreceptor survival. This prosurvival effect was most pronounced in the slowest degenerating mutants R562W and D670G. Images shown are representative for at least six different specimens for each genotype; DAPI (4',6-diamidino-2-phenylindole; blue/grey) was used as a nuclear counterstain.

the number of residual photoreceptor rows was decreased to $\sim 0.5$, indicating that at this stage likely only cone photoreceptors were remaining.

Further prolongation of the culture duration to P25 changed the picture considerably, to the point that no significant photoreceptor rescue could be detected in any of the four Pde6a mutants (Supplementary Figure S2). Only in the slowest degenerating D670G mutant did PJ34 treatment result in a minor, but statistically nonsignificant increase of photoreceptor survival.

This leaves us with a situation where the mutation-dependent rate of degeneration is reflected in the treatment outcomes. The most rapid degeneration (in the V685M model) could be treated to achieve an effect on TUNEL positivity in the short-term, 6 DIV treatment, but nothing else. The two medium rate degenerations (V685M*R562W, R562W) could be protected to the point of increased photoreceptor survival at both 6 and 10 DIV treatments, whereas the slowest degeneration (D670G) did the same, but in addition was the only one displaying a discernible numerical increase in surviving photoreceptor rows after treatment for 16 DIV. These results are summarized in Figure $4 a$, in which the treatment effects on photoreceptor survival are plotted against the treatment duration in the four Pde6a mutant genotypes. 

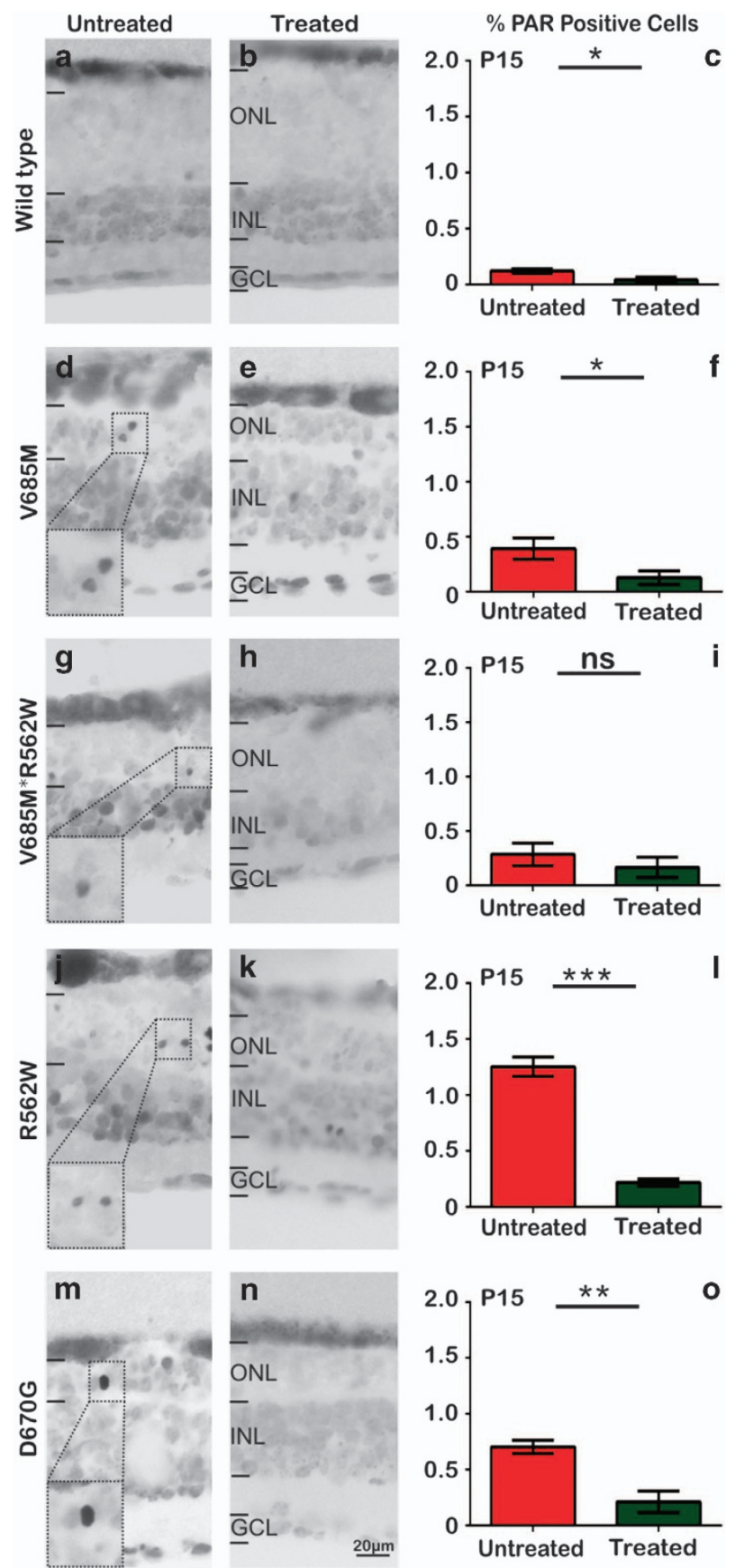

Figure 3. PARP inhibition decreases PAR accumulation in Pde6a mutant retina. In photoreceptors excessive PARP activity results in the accumulation of PAR. In retinal explant cultures, at P15, PARpositive cells were seen occasionally in the wt situation (a), yet with PJ34 treatment, resulting in a significant decrease of their numbers (b, quantification in c). A similar effect of PARP inhibition was seen in the V685M mutant (d-f), whereas in the V685M*R562W the reduction of PAR-positive cells did not attain statistical significance (g-i). The most pronounced effects of PARP inhibition were seen in the slowly degenerating R562W (j-I) and D670G (m-o) mutants. Images shown are representative of six different specimens per genotype.

In this condensed view, the treatment effects are exemplified by the areas between the curves representing treated and untreated situations.

Taken together, the results thus suggest that for each mutant situation there was a specific window of opportunity for successful treatment, the size of which depended on the strength of the genetic insult (Figure 4b).

\section{DISCUSSION}

To date, RP is still an untreatable condition. Major obstacles for the successful personalized therapy development are the insufficient understanding of cellular disease mechanisms and the enormous heterogeneity of disease-causing mutations. Our study addresses these two problems by showing that PARP activity is a common denominator in retinal degeneration caused by three different point mutations in the Pde6a gene. In addition to studying the effects of PARP inhibition on these three mutations in homozygous situations, we also investigated a recently generated compound heterozygous model, which is genotype-matched to a human subject suffering from RP. We show that in all four mutant situations PARP inhibition affords a significant photoreceptor protection, with the effect size determined by the time-point of therapeutic intervention and the severity of the genetically induced insult.

PDE6A mutations and cGMP signalling in retinitis pigmentosa

Human RP is characterized by a remarkable non-allelic genetic heterogeneity with around 60 different disease genes currently known (https://sph.uth.edu/Retnet; information retrieved in July 2015). Moreover, for each of these genes there are typically a large number of different disease-causing mutations, as is also true for the PDE6A gene for which at least 29 different mutations are known (http://www.hgmd.cf.ac.uk; information retrieved July 2015). In our attempts to create therapies that could act broadly on the various PDE6A RP genotypes, it is required that we increase our knowledge on if and how the degeneration processes differ between the latter. Several different mouse models carrying human homologous of Pde6a variants are known. ${ }^{13,15}$ In the present study, we took advantage of this and can now present mechanistic data on four such Pde6a mutation models, of which three are homozygous (V685M, R562W, and D670G), with the fourth being compound heterozygous (V685M*R562W).

Interestingly, the R562W, D670G, and V685M mutations all map to the catalytic domain of PDE6A. ${ }^{15}$ A previous study identified marked differences both in the expression of the different PDE6A mutant proteins and in the accumulation of cGMP, likely as a result of different consequences for the catalytic activity. In this respect, the V685M mutant showed the highest degree of cGMP accumulation, whereas the $\mathrm{V} 685 \mathrm{M}^{*} \mathrm{R} 562 \mathrm{~W}$ and the R562W were intermediate, and the D670G showed a comparatively weak cGMP accumulation. ${ }^{13}$ This variation in the strength of the genetic insults likely is the reason for the different disease progression phenotypes.

\section{PARP activity in retinal degeneration}

The activation of PARP is an important event during base excision repair of damaged DNA and PARP is sometimes addressed as the 'guardian of the genome'. ${ }^{16}$ Accordingly, PARP activity was found to have prosurvival effects in the inner ear, ${ }^{17}$ and, in mice, the absence of PARP reduces the overall lifespan. ${ }^{18}$ Furthermore, for modern cancer therapy PARP inhibition is proposed as an adjuvant to increase the sensitivity of cancer cells to genotoxic stress. ${ }^{19}$ PARP is also involved in the epigenetic regulation of gene expression via the PARylation of histone proteins, ${ }^{20}$ and possibly also via modifications on the DNA methylation pattern. ${ }^{21}$ Taken together, these actions of PARP might explain the extensive changes in DNA methylation ${ }^{22}$ and gene expression ${ }^{23}$ observed during photoreceptor degeneration.

An excessive activation of PARP is also frequently observed during cell death, including in neurodegenerative diseases. ${ }^{9,24,25}$ When we used an in situ PARP activity assay based on the 

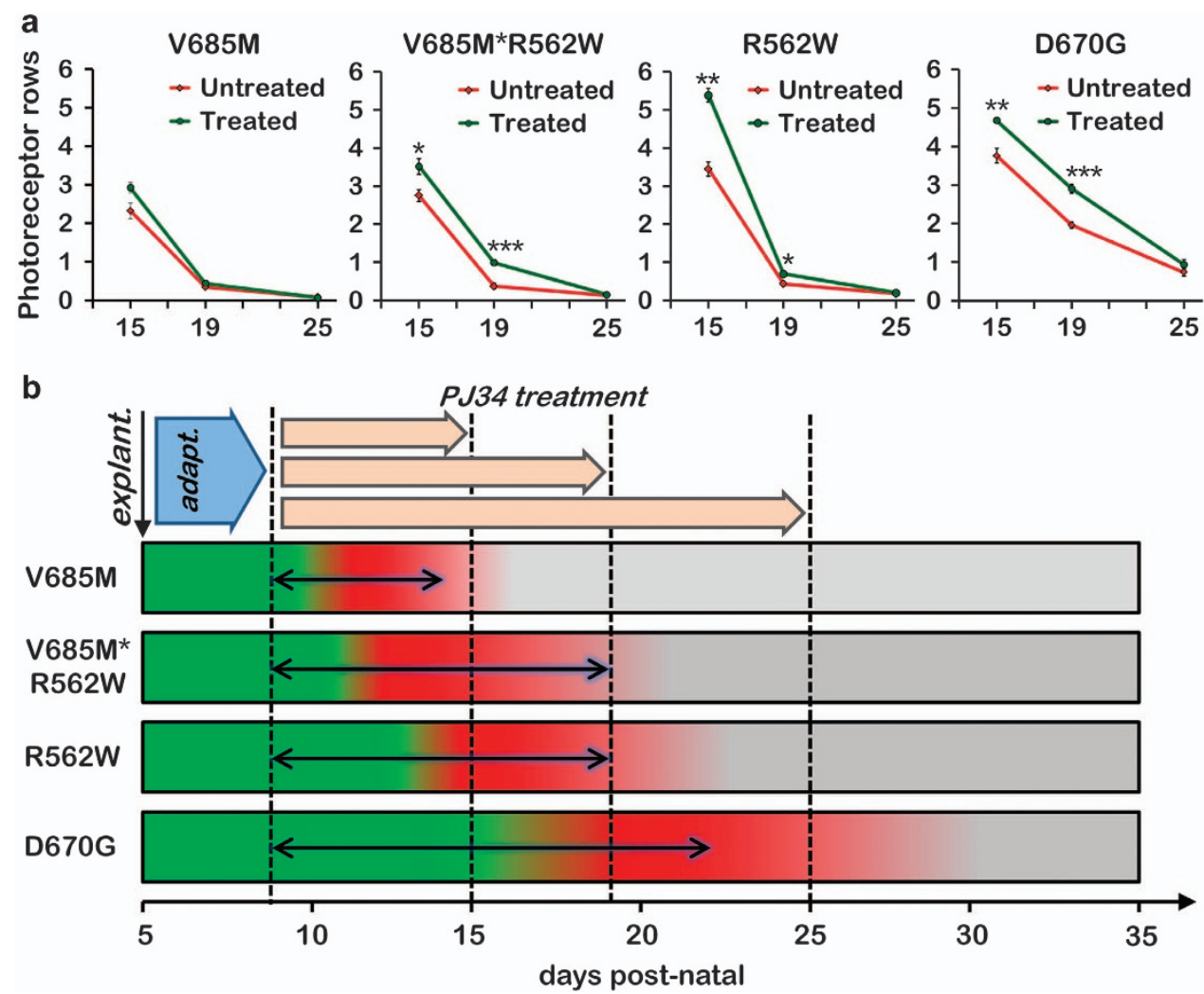

Figure 4. Treatment effects and windows of opportunity in different Pde6a mutants. When the number of surviving photoreceptor rows was plotted against the treatment duration, in the different genotypes, the effects of PJ34 treatment were variable with the V685M showing the smallest and the D670G mutant the biggest effect on photoreceptor survival (a). Retinas from Pde6a mutant animals were explanted at P5, left untreated to adapt to culture conditions for 4 days, and then treated with PJ34 until P15, P19, or P25. Initially, retinas appeared morphologically normal, healthy (illustrated here in green). During the degeneration period (red), ONL cells died until (virtually) all rod photoreceptors were lost (grey). The differences in treatment effects in Pde6a mutants corresponded with the onsets and speed of retinal degeneration, with V685M displaying the fastest and D670G the slowest disease progression. This resulted in differently sized windows of opportunity (black, double-headed arrows) with the slowest degenerating mutants displaying the largest window of opportunity (b).

incorporation of biotin-labelled $\mathrm{NAD}^{+},{ }^{26}$ we found that PARP activity was strongly increased in the photoreceptors of all four Pde6a mutant genotypes. This corresponds to earlier findings in the Pde6b mutant $r d 1$ and $r d 10$ mouse models for $\mathrm{RP},{ }^{10,11}$ and is also seen in many other animal models for RP, carrying diseasecausing mutations in a variety of different genes. ${ }^{11,27}$ As most of these models also display increased photoreceptor cGMP, it is possible that this rise is a component of the PARP activation. How this would occur is not fully understood yet, although previous works have suggested a sequential activation of protein kinase $G^{28}$ and histone deacetylase just before PARP activation. ${ }^{29}$

How could excessive PARP activity cause photoreceptor cell death? One possibility is that the excessive PARP-induced consumption of $\mathrm{NAD}^{+}$leads to a depletion of energy-containing substrates, such as ATP, and thus causes an energetic collapse. An alternative possibility might be that excessive accumulation of certain PAR species could be toxic to the cell. ${ }^{30}$ In this context, it is interesting to note that the TUNEL assay marks cells that have already undergone a strong fragmentation of the DNA. ${ }^{31,32}$ The fact that PARP activity and TUNEL assays partly colocalize in the same photoreceptor cells ${ }^{10}$ thus suggests that excessive PARP activation may have contributed to DNA damage. We noted that the proportions of PARP activity- and TUNEL-positive cells differed between the models (Figure 1), in that during rapid degeneration there was a much higher percentage of TUNEL-positive compared with that in PARP activity-positive cells, whereas during slow degeneration the percentages were about equal. Whether this indicates a situation-dependent difference in execution times of the two processes remains to be studied.

PARP inhibition as a therapeutic strategy to prevent RP

Although PARP activity and its role in DNA repair in general is seen as beneficial, ${ }^{18}$ in postmitotic neurons the demand for DNA repair is much lower, and hence under normal, physiological conditions, PARP inhibition or even genetic deletion has essentially no effect. ${ }^{9,33}$ However, under pathophysiological conditions, the aberrant and excessive PARP activation can be rectified by PARP inhibition, which would propose suitability in the RP situation.

The fact that PARP activity and also the accumulation of PAR colocalize with TUNEL ${ }^{10}$ may indicate that in the cascade of events leading to photoreceptor cell death, PARP activity is a relatively late event. While in general terms the targeting of late events may be less advantageous, in the case of PARP, the fact that many different mutations all cause PARP activity ${ }^{11}$ suggests that different cell death processes may converge on PARP as something like a late-stage common denominator. This makes PARP an attractive target for therapeutic interventions, as in diseases with a very high genetic heterogeneity, such as RP, this kind of therapy would be applicable to many different disease forms at once.

In the fastest degenerating V685M mutant, PJ34 treatment achieved only a relatively low degree of photoreceptor neuroprotection at P15, yet in the slowest degenerating D670G mutant, PJ34 treatment increased photoreceptor survival even at P25. Hence, in the different Pde6a mutants studied here the time 
window for therapeutic interventions was dependent on the strength of the genetically induced insult (Figure 4b). This provides a strong case for a personalized therapeutic approach, where the exact mutation will convey information with respect to how long the time window is open for PARP-centred interventions, and perhaps also other types of treatments.

Even when the correct window of opportunity was chosen (i.e. a treatment time-point and duration that showed an effect), PJ34 treatment did not halt retinal degeneration completely, in any of the four Pde6a mutants. While this could be due to insufficient inhibitory potential of PJ34 or even off-target effects, ${ }^{34}$ it could also be due to the concurrent activation of PARPindependent cell death pathways. ${ }^{11,35}$ In the latter scenario, the blocking of PARP activity would slow down the degeneration but could not ultimately prevent it. However, it is worth mentioning that even a comparatively minor delay in the progression of retinal degeneration in a rodent could potentially translate to many years of useful vision in human RP patients. ${ }^{36}$ In the current situation, where no treatment is available, this would already constitute a major progress in the development of medicines for rare diseases.

\section{CONCLUSION}

This is the first report demonstrating an excessive activation of PARP in photoreceptors suffering from different point mutations in the Pde6a gene. Taken together with earlier data obtained in several other RP animal models, ${ }^{11}$ this adds further weight to the notion that PARP is a common denominator in photoreceptor cell death and hence a promising target for therapy development.

Homozygous V685M and compound heterozygous V685M/ R562W Pde6a mutants used in this study were genotype-matched to specific forms of human PDE6A RP. Our study suggests that the strength of the insult caused by these mutations correlates with onset and progression of disease. This likely bears implications for any future personalized therapeutic intervention as the severity and differential character (homozygous versus compound heterozygous) of a mutation may affect efficacy of treatment, highlighting the need to identify the right treatment durations, analysis time-points, and animal models for future genotypematched therapy.

In conjunction with a careful clinical assessment of human RP phenotypes, our observations may impact the future design of clinical studies; for instance, in helping to define which genotype to include, at what time-point in the disease course, and what magnitude of treatment effect to expect.

\section{MATERIALS AND METHODS}

\section{Experimental animals}

$\mathrm{C} 3 \mathrm{H}$ wt mice ${ }^{37}$ were obtained from the Tuebingen university in-house animal facility. Homozygous mice carrying the V685M ( $\mathrm{nmf282}$ ) and D670G (nmf363) mutations were obtained from Jackson Labs (Bar Harbor, MA, USA). ${ }^{15}$ The R562W mutant was generated recently by GenOway (Lyon, France). ${ }^{13}$ Compound heterozygous V685M*R562W mutants were generated at the Tuebingen University by cross-breeding the respective homozygous mutants.

Animals had free access to food and water, were housed under standard $12 \mathrm{~h}$ lighting conditions, and were used irrespective of gender. Pde6a mice were killed at different time-points for histology (P9-P35) or for retinal explant culture (P5). All procedures were approved by the Tuebingen University committee on animal protection and performed in compliance with the ARVO statement for the use of animals in Ophthalmic and Visual Research. Protocols compliant with Section 4 of the German law on animal protection were reviewed and approved by the 'Einrichtung fur Tierschutz, Tierarztlichen Dienst und Labortierkunde' (Notifications 16.06 .12 and 01.06.14). All efforts were made to minimize the number of animals used and their suffering.
Retinal explant cultures

Mice were killed using decapitation at P5, and their heads were cleaned with $70 \%$ ethanol. The eyes were removed under sterile conditions, and subsequently incubated in $0.12 \%$ Proteinase $\mathrm{K}$ at $37^{\circ} \mathrm{C}$ for $5 \mathrm{~min}$. They were then washed with basal R16 medium containing $10 \%$ FCS for 2-3 min to inactivate Proteinase K. After that, the cornea, sclera, and lens were removed, so that only neural retina and retinal pigment epithelium (RPE) remained. The retinas were cut into four wedges and transferred onto the culturing membrane (Millicell, no. PIHA03050; Millipore, Cork, Ireland) with the RPE facing the membrane. The retinas were cultured in R16 medium with supplements ${ }^{38}$ for 4 days without treatment to adapt to in vitro conditions. From P9 onwards they were treated with $6 \mu \mathrm{M}$ PJ34 until P15 (6 days of treatment), P19 (10 days of treatment), or P25 (16 days of treatment). During the culturing period, the R16 medium was changed every 2 days and cultures were terminated using 4\% paraformaldehyde (PFA; Polysciences, Warrington, PA, USA) in $0.1 \mathrm{M}$ phosphate buffer ( $\mathrm{pH} 7.4)$ for $30 \mathrm{~min}$ at room temperature.

\section{Retinal tissue preparations and TUNEL assay}

The animals were killed by decapitation; the eyes were immediately enucleated, and directly embedded in Tissue-Tek cryomatrix (Leica, Bensheim, Germany) to obtain unfixed, frozen tissue for later use in the PARP activity assay.

To obtain fixed retinal preparations for PAR immunohistochemistry, the eyes were fixed with 4\% PFA in $0.1 \mathrm{M}$ phosphate buffer $(\mathrm{pH}$ 7.4) for $30 \mathrm{~min}$ at room temperature. After fixation, eyes were washed with PBS for $10 \mathrm{~min}$ and cryoprotected by incubation in graded sucrose solutions $(10 \%, 20 \%$, and $30 \%)$. Subsequently, tissues were embedded in cryomatrix and vertical sections $(12 \mu \mathrm{m})$ were obtained on a Leica CM3050S Microtome (Leica Biosystems, Wetzlar, Germany), air dried at $37^{\circ} \mathrm{C}$ for $1 \mathrm{~h}$, and stored at $-20^{\circ} \mathrm{C}$ until use.

The TUNEL Cell Death Detection Kit (conjugated with either fluorescein or TMR; Roche Diagnostics GmbH, Mannheim, Germany) was used to detect photoreceptor cell death, and performed according to the manufacturer's instructions.

\section{PARP enzyme activity assay}

Unfixed cryosections from Pde6a mutants were incubated with an Avidin/ Biotin Blocking Kit (Vector Laboratories, Burlingame, CA, USA), followed by incubation at $37^{\circ} \mathrm{C}$ for $2 \mathrm{~h}$ in PARP reaction mixture containing $10 \mathrm{mM}$ $\mathrm{MgCl}_{2}, 1 \mathrm{mM} \mathrm{DTT}, 5 \mathrm{mM}$ biotinylated $\mathrm{NAD}^{+}$(Trevigen, Gaithersburg, MD, USA) in $100 \mathrm{mM}$ Tris buffer with $0.2 \%$ Triton $\mathrm{X}-100(\mathrm{pH} 8.0)$. Incorporated biotin was detected by binding to avidin conjugated with Alexa Fluor 488 (1:800, $1 \mathrm{~h}$ at room temperature; Life Technologies, Darmstadt, Germany). For controls, biotinylated $\mathrm{NAD}^{+}$was omitted from the reaction mixture (not shown, but see Paquet-Durand et al. ${ }^{10}$ ).

\section{PAR immunohistochemistry}

For the PAR staining, fixed cryosections were dried at $37^{\circ} \mathrm{C}$ for $1 \mathrm{~h}$, and washed with PBS for $10 \mathrm{~min}$ at room temperature. To reduce nonspecific background, quenching solution $\left(100 \mu \mathrm{l} 30 \% \mathrm{H}_{2} \mathrm{O}_{2}, 400 \mu \mathrm{l} \mathrm{MeOH}\right.$, $500 \mu \mathrm{l}$ PBST) was put on each section for $20 \mathrm{~min}$ at room temperature. Sections were incubated in blocking solution (10\% NGS, 0.1\% PBST) for $1 \mathrm{~h}$ at room temperature. Primary PAR antibody (1:200; no. ALX-804-220; Enzo Life Sciences, Loerrach, Germany) was applied overnight at $4{ }^{\circ} \mathrm{C}$. After washing three times with PBS for $1 \mathrm{~h}$, sections were incubated with secondary antibody (biotinylated goat anti-mouse 1:150; Vector Laboratories, Burlingame, CA, USA). This was followed by incubation with Vector $A B C$ Kit (Vector Laboratories) for $1 \mathrm{~h}$ in $A B C$ reaction mixture ( $1 \mu \mathrm{l}$ avidin solution, $1 \mu \mathrm{l}$ biotin solution, in $148 \mu \mathrm{l} \mathrm{PBS})$. After washing three times with PBS for $10 \mathrm{~min}$, sections were visualized under a microscope.

Microscopy, cell counting, and statistical analysis

Light and fluorescence microscopy was performed on a Z1 ApoTome Microscope equipped with a Zeiss Axiocam Digital Camera (Zeiss, Jena, Germany). Images were captured using Zeiss Axiovision 4.7 software (Zeiss) and representative pictures were taken from central areas of the retina. Adobe Photoshop CS3 (Adobe Systems Incorporated, San Jose, CA, USA) and Corel Draw X3 software (Corel Corporation, Ottawa, Canada) was used for image processing. 
For cell quantifications, pictures were captured on whole radial sections using the Mosaix mode in Axiovision 4.7. TUNEL, PARP activity, and PAR-labelled cells were counted manually. First, the average size of an ONL cell was determined by counting all DAPI-positive cells in 3-6 rectangular areas distributed randomly over the ONL. ${ }^{11,14}$ This was repeated for retinal sections obtained from five different animals and the ONL cell size averaged. The total number of cells was then determined by dividing ONL area through the average ONL cell size. The number of positive cells was then divided by the total number of ONL cells giving the percentage of positive cells. All data given represent the means and standard deviation from three sections each for each animal, obtained from at least three different animals.

Statistical comparisons between experimental groups were made using one-way ANOVA and Bonferroni's correction using Prism 5 for Windows (GraphPad Software, La Jolla, CA, USA). Values are given as mean \pm S.E.M. Levels of significance were: not significant (NS) $=P>0.05,{ }^{*}=P<0.05$, ${ }^{* *}=P<0.01$, and ${ }^{* * *}=P<0.001$.

\section{ABBREVIATIONS}

DIV, days in vitro; GCL, ganglion cell layer; INL, inner nuclear layer; PDE, phosphodiesterase; ONL, outer nuclear layer; $\mathrm{P}$, postnatal day(s); PARP, poly (ADP-ribose)polymerase; PAR, poly(ADP-ribose); RP, retinitis pigmentosa; TUNEL, terminal deoxynucleotidyl transferase dUTP nick-end labelling.

\section{ACKNOWLEDGEMENTS}

We thank B Wissinger and $\mathrm{N}$ Weissschuh for comments and critical reading of the manuscript, as well as K Masarini and N Rieger for skilful technical assistance. This work was supported by the centre for ophthalmology of the Second People's Hospital of Yunnan Province and the Fourth affiliated Hospital of Kunming Medical University, the Tistou and Charlotte Kerstan Foundation (RD-CURE and SAH001/2015), the German research council (Deutsche Forschungsgemeinschaft; DFG:PA1751/7-1 and SA3040/1-1), the Alcon Research Institute (ARI), the European Union (DRUGSFORD: HEALTH-F2-2012-304963), and the Werner Reichardt Centre for Integrative Neuroscience (CIN) of the University of Tuebingen (EXC 307).

\section{AUTHOR CONTRIBUTIONS}

$\mathrm{KJ}$ carried out the in vitro retinal explant culture studies, participated in the in vivo characterization of Pde6a mutants, and drafted the manuscript. AS participated in the in vitro studies, the TUNEL and immunostainings, and carried out parts of the analysis. EZ, MU, and PE participated in the design of the study. $\mathrm{PE}$ and $\mathrm{MU}$ participated in the analysis and interpretation, and helped to write the manuscript. FPD and AS conceived the study, participated in its design and coordination, and helped to write the manuscript. All authors read and approved the final manuscript.

\section{COMPETING INTERESTS}

The authors declare no conflict of interest.

\section{REFERENCES}

1 Kennan A, Aherne A, Humphries P. Light in retinitis pigmentosa. Trends Genet 2005; 21: 103-110.

2 Chizzolini M, Galan A, Milan E, Sebastiani A, Costagliola C, Parmeggiani F. Good epidemiologic practice in retinitis pigmentosa: from phenotyping to biobanking. Curr Genomics 2011; 12: 260-266.

3 Bayes M, Giordano M, Balcells S, Grinberg D, Vilageliu L, Martínez I et al. Homozygous tandem duplication within the gene encoding the beta-subunit of rod phosphodiesterase as a cause for autosomal recessive retinitis pigmentosa. Hum Mutat 1995; 5: 228-234.

4 Tsang SH, Gouras P, Yamashita CK, Kjeldbye H, Fisher J, Farber DB et al. Retinal degeneration in mice lacking the gamma subunit of the rod cGMP phosphodiesterase. Science 1996; 272: 1026-1029.

5 Dryja TP, Rucinski DE, Chen SH, Berson EL. Frequency of mutations in the gene encoding the alpha subunit of rod CGMP-phosphodiesterase in autosomal recessive retinitis pigmentosa. Invest Ophthalmol Vis Sci 1999; 40: 1859-1865.

6 Keeler CE. The Inheritance of a retinal abnormality in white mice. Proc Natl Acad Sci USA 1924; 10: 329-333.

7 Chang B, Hawes NL, Hurd RE, Davisson MT, Nusinowitz S, Heckenlively JR et al. Retinal degeneration mutants in the mouse. Vision Res 2002; 42: 517-525.
8 David KK, Andrabi SA, Dawson TM, Dawson VL. Parthanatos, a messenger of death. Front Biosci 2009; 14: 1116-1128.

9 Sahaboglu A, Tanimoto N, Kaur J, Sancho-Pelluz J, Huber G, Fahl E et al. PARP1 gene knock-out increases resistance to retinal degeneration without affecting retinal function. PLoS One 2010; 5: e15495.

10 Paquet-Durand F, Silva J, Talukdar T, Johnson LE, Azadi S, van Veen $T$ et al. Excessive activation of poly(ADP-ribose) polymerase contributes to inherited photoreceptor degeneration in the retinal degeneration 1 mouse. J Neurosci 2007; 27: 10311-10319.

11 Arango-Gonzalez B, Trifunović D, Sahaboglu A, Kranz K, Michalakis S, Farinelli P et al. Identification of a common non-apoptotic cell death mechanism in hereditary retinal degeneration. PLoS One 2014; 9: e112142.

12 Corton M, Blanco MJ, Torres M, Sanchez-Salorio M, Carracedo A, Brion M et al. Identification of a novel mutation in the human PDE6A gene in autosomal recessive retinitis pigmentosa: homology with the $\mathrm{nmf} 28 / \mathrm{nmf} 28$ mice model. Clin Genet 2010; 78: 495-498.

13 Sothilingam V, Garcia Garrido M, Jiao K, Buena-Atienza E, Sahaboglu A, Trifunović $\mathrm{D}$ et al. Retinitis pigmentosa: Impact of different Pde6a point mutations on the disease phenotype. Hum Mol Genet 2015; 24: 5486-5499.

14 Sahaboglu A, Tanimoto N, Bolz S, Garrido MG, Ueffing M, Seeliger MW et al. Knockout of PARG110 confers resistance to CGMP-induced toxicity in mammalian photoreceptors. Cell Death Dis 2014; 5: e1234.

15 Sakamoto K, McCluskey M, Wensel TG, Naggert JK, Nishina PM. New mouse models for recessive retinitis pigmentosa caused by mutations in the Pde6a gene. Hum Mol Genet 2009; 18: 178-192.

16 Tong WM, Cortes U, Wang ZQ. Poly(ADP-ribose) polymerase: a guardian angel protecting the genome and suppressing tumorigenesis. Biochim Biophys Acta 2001; 1552: 27-37.

17 Jaumann M, Dettling J, Gubelt M, Zimmermann U, Gerling A, Paquet-Durand F et al. cGMP-Prkg1 signaling and Pde5 inhibition shelter cochlear hair cells and hearing function. Nat Med 2012; 18: 252-259.

18 Piskunova TS, Yurova MN, Ovsyannikov Al, Semenchenko AV, Zabezhinski MA, Popovich IG et al. Deficiency in poly(ADP-ribose) polymerase-1 (PARP-1) accelerates aging and spontaneous carcinogenesis in mice. Curr Gerontol Geriatr Res 2008; 2008: 754190

19 Mariano G, Ricciardi MR, Trisciuoglio D, Zampieri M, Ciccarone F, Guastafierro T et al. PARP inhibitor ABT-888 affects response of MDA-MB-231 cells to doxorubicin treatment, targeting Snail expression. Oncotarget 2015; 6: 15008-15021.

20 Quenet D, El Ramy R, Schreiber V, Dantzer F. The role of poly(ADP-ribosyl)ation in epigenetic events. Int J Biochem Cell Biol 2009; 41: 60-65.

21 Caiafa P, Guastafierro T, Zampieri M. Epigenetics: poly(ADP-ribosyl)ation of PARP-1 regulates genomic methylation patterns. FASEB $J$ 2009; 23: 672-678.

22 Farinelli P, Perera A, Arango-Gonzalez B, Trifunovic D, Wagner M, Carell T et al. DNA methylation and differential gene regulation in photoreceptor cell death. Cell Death Dis 2014; 5: e1558.

23 Azadi S, Paquet-Durand F, Medstrand P, van Veen T, Ekström PA. Up-regulation and increased phosphorylation of protein kinase $C$ (PKC) delta, mu and theta in the degenerating rd1 mouse retina. Mol Cell Neurosci 2006; 31: 759-773.

24 Wang Y, Kim NS, Li X, Greer PA, Koehler RC, Dawson VL et al. Calpain activation is not required for AIF translocation in PARP-1-dependent cell death (parthanatos). J Neurochem 2009; 110: 687-696.

25 Eliasson MJ, Sampei K, Mandir AS, Hurn PD, Traystman RJ, Bao J et al. Poly(ADP-ribose) polymerase gene disruption renders mice resistant to cerebral ischemia. Nat Med 1997; 3: 1089-1095.

26 Ekstrom PA, Ueffing M, Zrenner E, Paquet-Durand F. Novel in situ activity assays for the quantitative molecular analysis of neurodegenerative processes in the retina. Curr Med Chem 2014; 21: 3478-3493.

27 Kaur J, Mencl S, Sahaboglu A, Farinelli P, van Veen T, Zrenner E et al. Calpain and PARP activation during photoreceptor cell death in $\mathrm{P} 23 \mathrm{H}$ and $\mathrm{S} 334$ ter rhodopsin mutant rats. PLoS One 2011; 6: e22181.

28 Paquet-Durand F, Hauck SM, van Veen T, Ueffing M, Ekström P. PKG activity causes photoreceptor cell death in two retinitis pigmentosa models. J Neurochem 2009; 108: 796-810.

29 Sancho-Pelluz J, Alavi MV, Sahaboglu A, Kustermann S, Farinelli P, Azadi S et al. Excessive HDAC activation is critical for neurodegeneration in the rd1 mouse. Cell Death Dis 2010; 1: 1-9.

30 Andrabi SA, Dawson TM, Dawson VL. Mitochondrial and nuclear cross talk in cell death: parthanatos. Ann NY Acad Sci 2008; 1147: 233-241.

31 Gavrieli Y, Sherman Y, Ben-Sasson SA. Identification of programmed cell death in situ via specific labeling of nuclear DNA fragmentation. J Cell Biol 1992; 119: 493-501.

32 Grasl-Kraupp B, Ruttkay-Nedecky B, Koudelka H, Bukowska K, Bursch W, SchulteHermann $\mathrm{R}$ et al. In situ detection of fragmented DNA (TUNEL assay) fails to discriminate among apoptosis, necrosis, and autolytic cell death: a cautionary note. Hepatology 1995; 21: 1465-1468. 
33 Wang ZQ, Stingl L, Morrison C, Jantsch M, Los M, Schulze-Osthoff $\mathrm{K}$ et al. PARP is important for genomic stability but dispensable in apoptosis. Genes Dev 1997; 11: 2347-2358.

34 Antolin AA, Jalencas X, Yélamos J, Mestres J. Identification of pim kinases as novel targets for PJ34 with confounding effects in PARP biology. ACS Chem Biol 2012; 7: 1962-1967.

35 Trifunovic D, Sahaboglu A, Kaur J, Mencl S, Zrenner E, Ueffing M et al. Neuroprotective strategies for the treatment of inherited photoreceptor degeneration. Curr Mol Med 2012; 12: 598-612.

36 Quinn R. Comparing rat's to human's age: how old is my rat in people years? Nutrition 2005; 21: 775-777.

37 Sanyal S, Bal AK. Comparative light and electron microscopic study of retinal histogenesis in normal and rd mutant mice. Z Anat Entwicklungsgesch 1973; 142 219-238.
38 Caffe AR, Ahuja P, Holmqvist B, Azadi S, Forsell J, Holmqvist I et al. Mouse retina explants after long-term culture in serum free medium. J Chem Neuroanat 2001; 22: $263-273$.

This work is licensed under a Creative Commons Attribution 4.0 International License. The images or other third party material in this article are included in the article's Creative Commons license, unless indicated otherwise in the credit line; if the material is not included under the Creative Commons license, users will need to obtain permission from the license holder to reproduce the material. To view a copy of this license, visit http://creativecommons.org/licenses/ by/4.0/

(c) The Author(s) 2016

Supplementary Information accompanies the paper on the Cell Death and Discovery website (http://www.nature.com/cddiscovery) 